\title{
Design of onboard vehicle diagnosis fault monitoring system using IOT and Machine learning
}

\author{
Anil N S 1 , Hari Charan Reddy ${ }^{2}$, Kunal Gaurav ${ }^{3}$, Navya $\mathbf{R}^{4}$ \\ Associate Professor, Dept. of ECE, East West Institute of Technology, Bangalore, India ${ }^{1}$ \\ B.E Student, Dept. of ECE, East West Institute of Technology, Bangalore, India ${ }^{2,3,4}$
}

\begin{abstract}
Internet of Vehicles is an upcoming technology. In our project, we are taking a step towards this On-board diagnostics (OBD) is a simple vehicle self-diagnosing system and have reporting capability. OBD systems give the vehicle owner or repair technician access to the status of the variety of vehicle subsystems. The amount of diagnostic information available via OBD has varied broadly since its introduction in the early 1980s versions of on-board vehicle computers. With the help of OBD II engine parameters like engine oil temperature, run time since engine start, engine coolant temperature etc are monitored. Through this, the status of the vehicle is diagnosed by the OBD system and the report is transferred using Wi-Fi to the car owner's Smartphone. Using the system, additional sensors can be added to a vehicle to upgrade its functionality, which the system supports. Additionally, driver assist functions are introduced, helping the driver during travel.
\end{abstract}

Keywords: autonomous vehicles, IOT, machine learning, predictive model, prediction of the failure.

\section{INTRODUCTION}

When driving your vehicle, glancing at your dashboard, have you ever thought of collecting the meter readings and do some analysis? These data may contain hidden treasures. For individuals, it can reflect your driving habits; it can tell you your speed, your average mpg, how many traffic lights you have, and your waiting time at each cross. For companies, these data are critical for real-time monitoring in fleet management. Vehicle condition, work load distribution, gasoline efficiency, and even vehicle location can all be fed back to a central control system through cloud. Companies can use machine learning to feed the data into a training model to predict the cost and even analyze the driver's characteristics. As IoT is widely spreading, the above applications won't be far away. With the Arduino MKR boards, targeting at IoT applications, you can build a device that talks to your car and uploads telemetric data into cloud all by yourself. Isn't that cool?

Internet of Vehicles is an upcoming technology. In our project, we are taking a step towards this On-board diagnostics (OBD) is a simple vehicle self-diagnosing system and have reporting capability. OBD systems give the vehicle owner or repair technician access to the status of the variety of vehicle subsystems. The amount of diagnostic information available via OBD has varied broadly since its introduction in the early 1980s versions of on-board vehicle computers. With the help of OBD II engine parameters like engine oil temperature, engine RPM, throttle position, run time since engine start, engine coolant temperature etc are monitored.

\section{ON BOARD DIAGNOSTIC SYSTEM}

This project demonstrates the architecture of an IOT prototype platform for On-Board Diagnostic System (OBD) and a IOT based application. Driver is also provided with a user interface through which user can read any of the sensor values by selecting on the list. Diagnosis of faults in parameters, notification of sudden changes to the driver, and indication regarding the reason for the fault are included in the system setup. The driver can find out the faults in car on the spot and this does not require any other tool for it. If in case the vehicle got struck in remote areas, the proposed OBD system inside the vehicle can be connected to the vehicle service center by providing internet facility. We are implementing this project on Arduino microcontroller. The advancement in cloud computing along with internet of things (IOT) has given a promising opportunity to resoluteness the challenges caused by the expanding transportation issues. It presents a novel multilayered vehicular data cloud platform by using cloud computing and IOT technologies. The two innovative vehicular data cloud services, an intelligent parking cloud service and a vehicular data excavation cloud service, for vehicle warranty analytic thinking in the IOT environment are also presented. 


\section{IJIREEICE}

\section{International Journal of Innovative Research in Electrical, Electronics, Instrumentation and Control Engineering}

Vol. 9, Issue 8, August 2021

\section{DOI 10.17148/IJIREEICE.2021.9802}

The automotive engine contains different system of rules that operate to fulfill the continuous function of the combustion. To insure un-interrupted function and optimal performance, various types of condition monitoring technologies are being used with different advanced data processing techniques. Developing an embedded system for detecting the vehicle condition by monitoring the internal parameters that are used in evaluating the vehicles electric current health condition. Traveler information dramatic plays a critical role in supporting refuge, security, mobility, and in improving the reliableness of travel. This traveler information can be a continuous data on performance of the vehicle and the position of its internal elements. An in-vehicle embedded organization is being developed to generate a vehicle health report (VHR) whenever needed by the user. It also acts as an eco friendly vehicle by monitoring the emissions from the car which in turn avail in regulating (by taking proper actions to reduce the emissions as per the issues shown within VHR) the environmental pollution. It predicts the future errors so that the driver can have a continuous travel and can avoid accident. The data required for generating the health report consists of parameter values (outputs of in-built sensor) of different systems inside the vehicle. This data can be obtained using the OBD-II protocol which is followed by the vehicle manufactured after 1996. It uses Lab VIEW as platform that has automotive diagnostic command set tool kit which helps in building up the software required to communicate with the vehicle's ECU through OBD-II system. OBD-II technology has been created to diagnose Vehicle‘s condition.

\section{PROBLEM STATEMENT}

The OBD system requires a scan tool which is necessary for its working. This scan tool has to be plugged in to the port provided for it within the vehicle.

The communication between the vehicle ECUs and scan tool is by sending trouble codes. Each trouble code has a particular parameter associated with it and this database of trouble codes will be provided by the manufacturer. Whenever you feed a trouble code, the system replies back with its associated value. The system was designed such that whenever a fault occurs, it reports to the user. The user could not know where the fault has been occurred in the system. There are certain limitations with this system such as

-Scan tool is necessary.

-Needs a technician to diagnose faults.

-Time required for updating software is comparatively high.

The proposed design for the OBD system uses internet interface instead of traditional kit and it does not need a scan tool for diagnosis. This system will directly inform the exact place of fault occurrence in the vehicle to the user without the need of trouble codes. Hence we can diagnose on his own without the help of technicians and service centre. Vehicle systems are complex both in hardware and software so their maintenance is challenging. Maintenance strategy being used in vehicle industry is normally reactive that results in reduction of lifetime of vehicle and also loss of money.

\section{METHODOLOGY}

Methodology gives the systematical, theoretical analysis of the methods applied to develop and maintain onboard vehicle diagnosis fault monitoring system using IOT and Machine learning. The aim of the work is to create software tools that allow you to control the technical condition of the car and predict the failure of individual units.

\section{Hardware Requirements and Software Requirement:}

Arduino Uno is a microcontroller board based on the ATmega328P. It has 14 digital input/output pins (of which 6 can be used as PWM outputs), 6 analog inputs, a $16 \mathrm{MHz}$ quartz crystal, a USB connection, a power jack, an ICSP header and a reset button. It contains everything needed to support the microcontroller; simply connect it to a computer with a USB cable or power it with an AC-to-DC adapter or battery to get started. A liquid-crystal display (LCD) is a flat-panel display or other electronically modulated optical device that uses the light-modulating properties of liquid crystals. Liquid crystals do not emit light directly, instead using a backlight or reflector to produce images in color or monochrome. The human ear can hear sound frequency around 20HZ $\sim 20 \mathrm{~K} \mathrm{HZ}$, and ultrasonic is the sound wave beyond the human ability of $20 \mathrm{~K} \mathrm{HZ}$. An ultrasonic sensor is an instrument that measures the distance to an object using ultrasonic sound waves. An ultrasonic sensor uses a transducer to send and receive ultrasonic pulses that relay back information about an object's proximity.The LM35 series are precision integrated-circuit temperature sensors, whose output voltage is linearly proportional to the Celsius (Centigrade) temperature. The LM35 thus has an advantage over linear temperature sensors calibrated in ${ }^{\circ}$ Kelvin, as the user is not required to subtract a large constant voltage from its output to obtain convenient Centigrade scaling. An accelerometer is a sensor that measures the physical acceleration experienced by an object due to inertial forces or due to mechanical excitation. An accelerometer behaves as a damped mass on a spring. When the accelerometer experiences acceleration, the mass is displaced and the 


\section{International Journal of Innovative Research in Electrical, Electronics, Instrumentation and Control Engineering}

Vol. 9, Issue 8, August 2021

\section{DOI 10.17148/IJIREEICE.2021.9802}

displacement is then measured to give the acceleration. ESP8266 offers a complete and self-contained Wi-Fi net working solution, allowing it to either host the application or to off load all Wi-Fi net working functions from another application processor. When ESP8266 hosts the application, and when it is the only application processor in the device, it is able to boot up directly from an external flash. A photoresistor (also known as a light-dependent resistor, LDR, or photo-conductive cell) is a passive component that decreases resistance with respect to receiving luminosity (light) on the component's sensitive surface. The resistance of a photoresistor decreases with increase in incident light intensity; in other words, it exhibits photoconductivity. A photoresistor can be applied in light-sensitive detector circuits and light-activated and dark-activated switching circuits acting as a resistance semiconductor.

\section{DESIGN AND IMEPLEMENTAION}

The replacement of diagnosis Tool kit with Automotive IOT provides lesser time for updating of ECU data and it is possible to use Wi-Fi for networking other domains of the vehicle. The proposed method of diagnosing the vehicle is different from the existing OBD systems. As stated earlier, this system does not require a scan-tool, technician and service centers. This follows the server-client architecture, as cloud being the server. The different parts of the vehicle are connected with multiple ECUs and these ECUs will have sensors attached with it for monitoring.

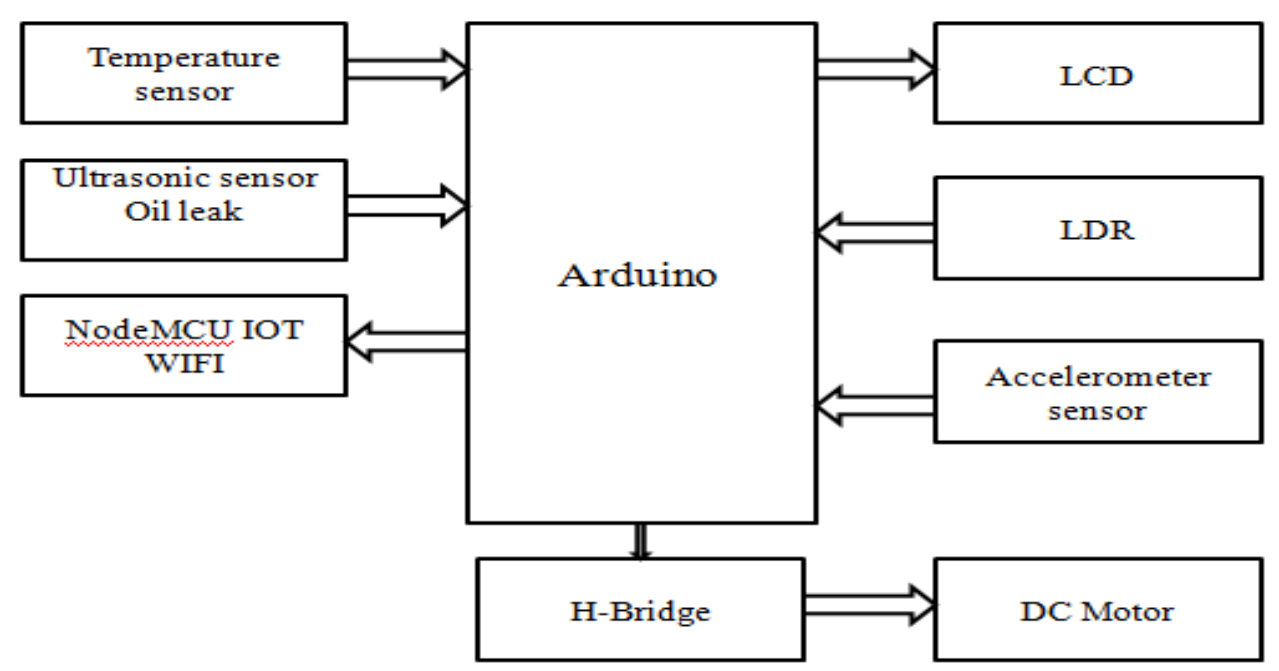

Fig. 5.1 Block diagram

All ECUs will be connected to the server and it will be collecting values from the ECUs. The user can view any realtime value of the parameter just by using mobile phone. So the user knows the condition and performance of the vehicle when driving.

Software development

To develop the software package, the Python programming language will be used, the development environment will be the Eclipse editor. To determine the dependencies between attributes, ridge regression is used. This is one of the methods of reducing the dimension. Often it is used to combat data redundancy when independent variables correlate with each other. The consequence of this is the poor conditioning of the matrix and the instability of the estimates of the regression coefficients. The use of ridge regression is often justified by the fact that this is a practical technique, with it you can, if desired; obtain a lower mean square error. Lasso (Least absolute shrinkage and selection operator) a method for estimating the coefficients of a linear regression model. 
International Journal of Innovative Research in Electrical, Electronics, Instrumentation and Control Engineering

Vol. 9, Issue 8, August 2021

DOI 10.17148/IJIREEICE.2021.9802

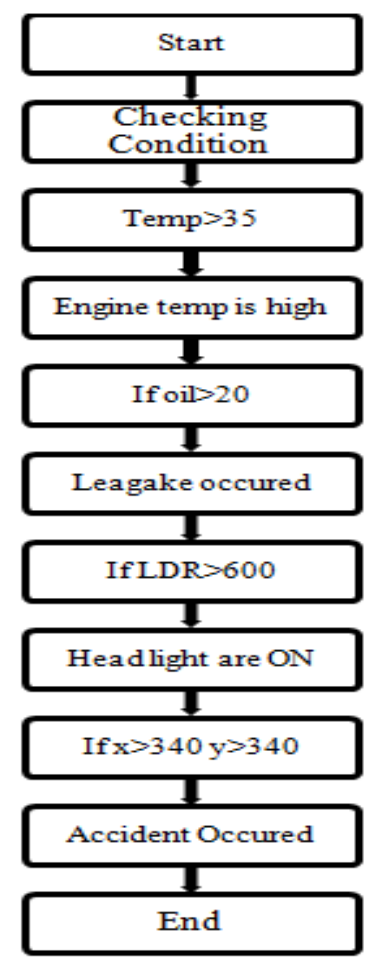

Fig. 5.2 Block diagram

In this project, the Atmega328 controller has been used with some other sensors. The main aim of the project is to get to know the actual problem of the vehicle. So in this project, the LM35 temperature sensor has been used to check the engine temperature and ultrasonic sensor is used to check the level of oil. So all this data has been sent to the concerned person. Also, the accelerometer sensor is also been used so that if the vehicle has met the accident and LDR is used for turning ON/OFF the lights of the vehicle and all the data has been sent to the concerned person. Start, it represents the start point of OBD system. Checking the condition: To configure checking of a condition, by using various sensors. If temperature is greater than $35^{\prime} \mathrm{C}$, then it give person a message that engine temperature is higher or more and give message to stop the cars. If temperature is less than $35^{\prime} \mathrm{C}$, then it give a person a message that engine is normal. If oil leakage is occur it is detect by ultrasonic sensor then it collect the information and send it to the person a message that leakage is occurred stop the vehicle. If oil leakage is not occurred, then it sends a person message that no leakage is occurred. If LDR is greater than $600 \mathrm{ohms}$ then it give a person message that light in a vehicle is ON please switched it OFF.

If LDR is less than $600 \mathrm{ohms}$ then it give person a message that light in a vehicle is OFF. Accelerometer sensor is used to detect the damaged occur during driving or car parking and it send the message to the concern person.

\section{RESULTS}

The OBD model currently performs functions such as checking temperature, oil leakage, and light in vehicle and bumper crashes. The OBD model movement is achieved by dc motors controlled by software modules.

As a result, we get a forecast of events in the near future, the shorter the time window for the forecast we need, the more accurate the result of the operation of neural networks, however, with proper configuration of the input data array and more accurate triggers, you can achieve a long-term forecast, the fastest of which is heating cycles, since no problems were found in the operation of the car, the long-term of simple analyzes can be considered the prediction of the failure of the car catalyst. 


\section{IJIREEICE}

\section{International Journal of Innovative Research in Electrical, Electronics, Instrumentation and Control Engineering}

Vol. 9, Issue 8, August 2021

DOI 10.17148/IJIREEICE.2021.9802

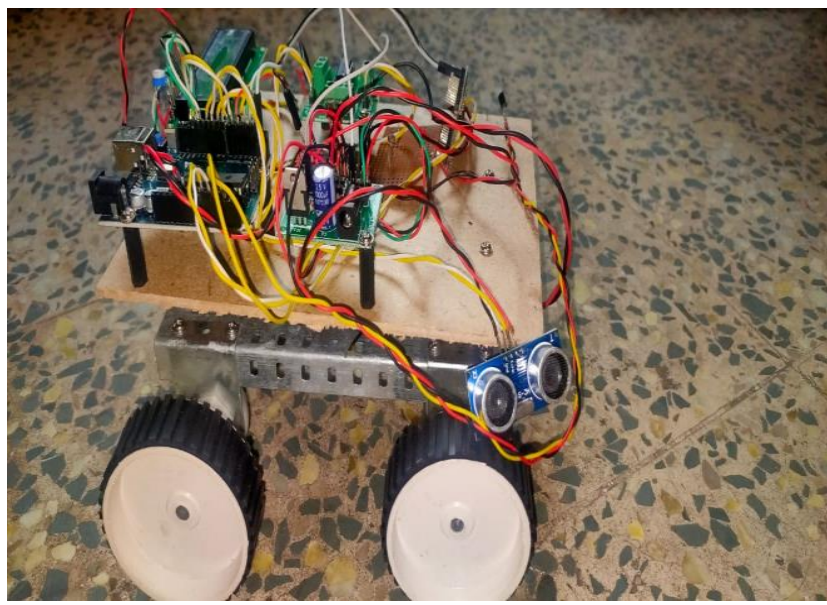

Fig. 6 Image of OBD project

\section{CONCLUSION}

As a result, Multipurpose autonomous on board vehicle diagnosis module has successfully implemented and tested for various functions like checking temperature of engine, oil leakage, light in vehicle, and bumper crashes. The results of this project are to find appropriate features that can identify fault in vehicle. we get a forecast of events in the near future, the shorter the time window for the forecast we need, the more accurate the result of the operation of neural networks, however, with proper configuration of the input data array and more accurate triggers, you can achieve a long-term forecast, the fastest of which is heating cycles, since no problems were found in the operation of the car, the long-term of simple analyzes can be considered the prediction of the failure of the car catalyst.

\section{REFERENCES}

[1] Mario Gerla, Eun-Kyu Lee, Giovanni Pau, "Internet of Vehicles: From Intelligent Grid to Autonomous cars and Vehicular Clouds", IEEE World Forum on Internet of Things, 2014

[2] Abhinyaa Balasundaram, Aiswarya Udayakumar, Abdul Muthalip , "IoT Based Vehicle Emission Monitoring System”, National Conference on Networks, Intelligence and Computing Systems, IJIRST, March 2017.

[3] Mohammad Rubaiyat Tanvir Hossain, Md.Asif Shahjalal, Nowroz Farhan Nur. "Real-time vehicle monitoring and traffic management" Cox'z Bazar, Bangladesh, February 16-18, 2017.

[4] Minghe Yu, Dapeng Zhang, Yurong Cheng, Mingshaun Wang. "Real-time vehicle monitoring and traffic management". Control and Decision Conference (CCDC), 2011 Chinese, 4192-4197.

[5] Sagar Sukode, Shilpa Gite. "Intelligent Transportation System with help of IR sensor". International Journal of Applied Engineering Research, ISSN 0973- 4562Volume 10, Number 8(2015), pp.19513-19523.

[6] M.G. Gorodnichev, A.E. Kochupalov, R.A. Gematudinov, "About some methods of rendering of text information in mobile applications", Quality. Innovations. Education, 2018, nNo. 6 (157), pp. 142-146.

[7] D.N. Suvorov, H.A. Dzhabrailov, "Influence of the temperature of the asphalt mixture on the quality of its seals", Automation and control in technical systems, 2014, no. 1-2 (9), pp. 165-171. 